\title{
Superconductivity in Heavy Alkaline-Earths Intercalated Graphites
}

\author{
J. S. Kim, ${ }^{1}$ L. Boeri, ${ }^{1}$ J. R. O'Brien, ${ }^{2}$ F. S. Razavi, ${ }^{3}$ and R. K. Kremer ${ }^{1}$ \\ ${ }^{1}$ Max-Planck-Institut für Festkörperforschung, Heisenbergstraße 1, D-70569 Stuttgart, Germany \\ ${ }^{2}$ Quantum Design, 6325 Lusk Boulevard, San Diego, California 92121, USA \\ ${ }^{3}$ Department of Physics, Brock University, St. Catharines, Ontario, L2S 3A1, Canada
}

(Dated: September 23, 2018)

\begin{abstract}
We report the discovery of superconductivity below 1.65(6) $\mathrm{K}$ in Sr-intercalated graphite $\mathrm{SrC}_{6}$, by susceptibility and specific heat $\left(C_{p}\right)$ measurements. In comparison with $\mathrm{CaC}_{6}$, we found that the anisotropy of the upper critical fields for $\mathrm{SrC}_{6}$ is much reduced. The $C_{p}$ anomaly at $T_{c}$ is smaller than the BCS prediction indicating an anisotropic superconducting gap for $\mathrm{SrC}_{6}$ similar to $\mathrm{CaC}_{6}$. The significantly lower $T_{c}$ of $\mathrm{SrC}_{6}$ as compared to $\mathrm{CaC}_{6}$ can be understood in terms of "negative" pressure effects, which decreases the electron-phonon coupling for both in-plane intercalant and the out-of-plane $\mathrm{C}$ phonon modes. We observed no superconductivity for $\mathrm{BaC}_{6}$ down to $0.3 \mathrm{~K}$.
\end{abstract}

PACS numbers: 74.70.Ad, 74.25.Bt, 74.62.Fj, 74.25.Kc

The discovery of superconductivity in $\mathrm{YbC}_{6}$ and $\mathrm{CaC}_{6}$ [1, 2] initiated intensive theoretical and experimental investigations $[3,4,5,6,7,8,9,10,11,12]$ on the alkaline-earth graphite intercalation compounds (GICs). The results of the experimental studies, specially the observation of a $\mathrm{Ca}$ isotope effect [12], strongly favor electron-phonon(e-ph) coupling rather than exotic electronic mechanisms [3]. Recent ab-initio electronic structure calculations prove that, in contrast to $\mathrm{MgB}_{2}, e-\mathrm{ph}$ coupling involving electronic interlayer (IL) states becomes relevant [3] and is sufficiently strong to generate the relatively high $T_{c}$ 's $[4,5,5]$. These findings stimulated discussions on the variability of the e-ph coupling strength in different branches of the electronic and vibrational states for honeycomb layered compounds like $\mathrm{MgB}_{2}, \mathrm{CaSi}_{2}$ [13], (Ca,Sr)AlSi [14] and also the hypothetical $\mathrm{Li}_{2} \mathrm{~B}_{2}$ [15].

Many issues still remain open, especially about the nature of the relevant phonons. In order to modify the phonon spectrum, Ca isotope substitution [12] and hydrostatic pressure experiments [8, 9, 10] have been performed. The isotope experiments reported a surprisingly high isotope exponent for $\mathrm{Ca}, \alpha(\mathrm{Ca}) \approx 0.5$, close to the BCS limit, suggesting a dominant role of the Ca phonons in the $e$-ph coupling [12]. However, ab-initio calculations predicted similar isotope exponents $\sim 0.25$ for $\mathrm{Ca}$ and $\mathrm{C}$, pointing to comparable contributions to the $e$-ph coupling from the $\mathrm{Ca}$ and $\mathrm{C}$ phonons [5]. The positive pressure dependence of $T_{c}$ found for $\mathrm{CaC}_{6}$ has been discussed in terms of phonon softening for the in-plane Ca vibrations [9, 16]. Although the experimentally determined $T_{c}$ 's grow almost linearly with pressure, the ab-initio calculations predicted a non-linear increase with a reduced magnitude [9]. To resolve these discrepancies, possible anharmonic effects of the ultrasoft intercalant phonon modes, or a continuous superconducting gap distribution due to anisotropic $e$-ph coupling have been suggested [17, 18], asking for further investigations.

Another way to modify the relevant phonon modes is to vary the intercalant species and replace $\mathrm{Ca}$ with other alkaline-earths such as $\mathrm{Sr}$ or Ba. Mazin pointed out that for $\mathrm{CaC}_{6}$ and $\mathrm{YbC}_{6}$ the square root of the mass ratio of the intercalants is only $15 \%$ larger than the ratio of their $T_{c}$ 's [4]. Thus, according to this "isotope" effect argument other alkaline-earths GICs may as well be superconducting. In fact, subsequent $a b$-initio calculations predicted superconductivity for $\mathrm{SrC}_{6}$ at $3.1 \mathrm{~K}$ and $\mathrm{BaC}_{6}$ at $0.2 \mathrm{~K}[16]$. In this Letter, we report the discovery of superconductivity in $\mathrm{SrC}_{6}$ at $T_{c}=1.65(6) \mathrm{K}$ by susceptibility and specific heat measurements and the absence of superconductivity in $\mathrm{BaC}_{6}$ down to $\sim 0.3 \mathrm{~K}$. The superconducting properties of $\mathrm{SrC}_{6}$ as well as the ab-initio calculations clearly demonstrate that $\mathrm{SrC}_{6}$ can serve to bridge the two seemingly different classes of the superconducting GICs: the low- $T_{c}$ alkali GICs and the newlydiscovered "high- $T_{c}$ " systems, $\mathrm{CaC}_{6}$ or $\mathrm{YbC}_{6}$. Furthermore, the comparison of $\mathrm{SrC}_{6}$ with $\mathrm{CaC}_{6}$ provides a better insight into the unconventional nature of superconductivity in alkaline-earth GICs.

Samples of $\mathrm{SrC}_{6}$ and $\mathrm{BaC}_{6}$ were synthesized from pieces of highly oriented pyrolytic graphite (Advanced Ceramics, size $\approx 3 \times 1 \times 1 \mathrm{~mm}^{3}$ ) and $\mathrm{Sr}(99.95 \%)$ or $\mathrm{Ba}$ $(99.95 \%)$ metal by a vapor phase reaction performed for more than a month at $470^{\circ} \mathrm{C}$ and $500^{\circ} \mathrm{C}$, respectively. Xray diffraction patterns show no reflections due to pristine graphite or other higher stage intercalated phases, indicating good sample homogeneity. The graphite layer distance increases from $\mathrm{CaC}_{6}(4.50 \AA)$ to $\mathrm{SrC}_{6}(4.95 \AA)$ and to $\mathrm{BaC}_{6}(5.25 \AA)$ as expected from the size of the intercalant atoms. The stacking sequence of $\mathrm{SrC}_{6}$ and $\mathrm{BaC}_{6}$ is found to be of the $\alpha \beta$-type (space group $\mathrm{P} 6_{3} / \mathrm{mmc}$ ) [19] which differs from the $\alpha \beta \gamma$-type stacking in $\mathrm{CaC}_{6}$. Superconductivity for $\mathrm{SrC}_{6}$ was first observed by ac magnetic susceptibility $(119 \mathrm{~Hz})$ measurements to $0.3 \mathrm{~K}$, and subsequently confirmed by specific heat measurements using a PPMS ${ }^{3} \mathrm{He}$ calorimeter (Quantum Design). The pressure dependence of $T_{c}$ was measured up to $\sim 1 \mathrm{GPa}$ as described in detail elsewhere [9]. In order to understand the 
superconducting properties of $\mathrm{SrC}_{6}$ and compare with those of $\mathrm{CaC}_{6}$, we also performed ab-initio calculations of the electron-phonon properties for $\mathrm{SrC}_{6}$ using the experimental $\alpha \beta$ stacking [20, 21].

Figure 1 shows a sharp superconducting transition in the magnetic susceptibility $\chi(T)$ at $T_{c}=1.65(6) \mathrm{K}$ in $\mathrm{SrC}_{6}$. The transition width $\Delta T_{c} \approx 0.06 \mathrm{~K}$ was determined as the temperature difference between $10 \%$ and $90 \%$ of the diamagnetic shielding. In a magnetic field, $T_{c}$ shifts to lower temperatures and the superconducting transition broadens. In $\mathrm{BaC}_{6}$, we cannot find any signature of superconductivity down to $0.3 \mathrm{~K}$.

The upper critical fields, $H_{\mathrm{c} 2}^{\|, \perp}$, for $H$ parallel and perpedicular to the $c$ axis follow the Werthamer-HelfandHohenberg (WHH) prediction rather well 23] (Fig. 1(b)). A slight deviation from the WHH curve is found at low temperatures, which has been observed in other GICs such as $\mathrm{CaC}_{6}$ and $\mathrm{KC}_{8}$ 24]. Within the scope of the WHH approximation, we obtained $H_{c 2}^{\|}(0) \approx 138$ Oe and $H_{c 2}^{\perp}(0) \approx 276$ Oe as well as the corresponding coherence lengths, $\xi_{a b}(0) \approx 1510 \AA$ and $\xi_{c}(0) \approx 700 \AA$. Similar to $\mathrm{CaC}_{6}, \xi_{c}(0)$ is larger than the graphite layer distance, indicating 3-dimensional (3D) superconductivity.

Bulk superconductivity in $\mathrm{SrC}_{6}$ is confirmed by specific heat $\left(C_{p}\right)$ measurements. The characteristic $C_{p}$ anomaly at $T_{c}=1.65 \mathrm{~K}$ is clearly observed at $H=0$, and completely disappears with $H=500$ Oe $\left(H>H_{\mathrm{c} 2}(0)\right)$. There is no offset of $C_{p} / T$ at $H=0$ as $T \rightarrow 0 \mathrm{~K}$, indicating a complete superconducting phase. Similar to $\mathrm{CaC}_{6}$, the normal state $C_{p}$ deviates slightly from a $T^{3}$ dependence (the inset of Fig. 22), due to low-lying Einstein phonon modes. The normal state $C_{p}$ can well be

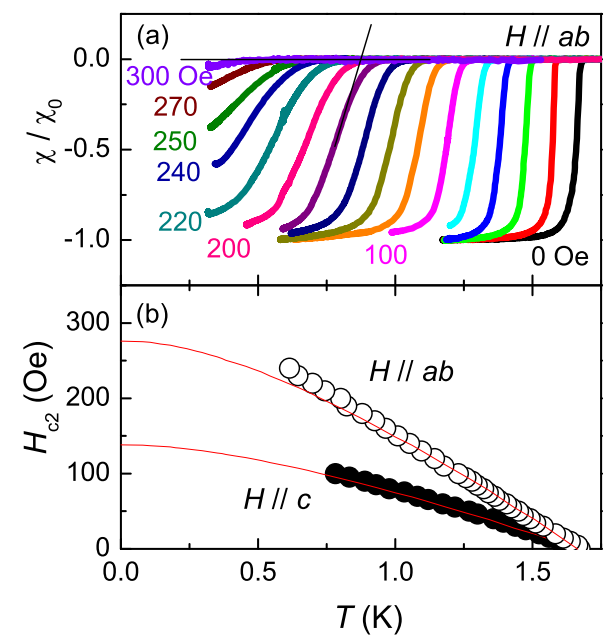

FIG. 1: (Color online) (a) Temperature dependence of the $a c$ susceptibility of $\mathrm{SrC}_{6}$ for various fields (as indicated) perpendicular to the $c$-axis. $T_{c}$ is determined from the intersection between the extrapolated lines of the steepest slope of $\chi(T)$ and of the normal state $\chi(T)$ as shown by the solid (black) lines. (b) The $H_{c 2}(T)$ for $H \| a b$ plane and $H \| c$ axis. The WHH curve for both $H$ directions are shown with (red) lines. described by $C_{p}(T)=\gamma_{N} T+C_{p h}(T)$ where $\gamma_{N}$ is the Sommerfeld constant, and $C_{p h}(T)=\beta T^{3}+\delta T^{5}$ is the lattice contribution. The best fit to the $H=500$ Oe data yields $\gamma_{N}=5.92(1) \mathrm{mJ} / \mathrm{mol} \mathrm{K}^{2}, \beta=0.191(1) \mathrm{mJ} / \mathrm{mol}$ $\mathrm{K}^{4}$, and $\delta=0.801(4) \mu \mathrm{J} / \mathrm{mol} \mathrm{K}^{6}$. The estimated Debye temperature $\Theta_{D}(0)=414(1) \mathrm{K}$ is lower than that of $\mathrm{CaC}_{6}\left(\Theta_{D}(0)=598 \mathrm{~K}\right)$ as expected from the atomic mass difference. From a comparison of $\gamma_{N}$ with the calculated density of states at the Fermi level $E_{F}, N(0)$, we estimate the $e$-ph coupling strength $\lambda$ using the relation $\gamma_{N}=$ $\left(2 \pi^{2} k_{B}^{2} / 3\right) N(0)(1+\lambda)$. With $N(0)=1.63$ states $/ \mathrm{eV} \cdot$ cell, we arrive at $\lambda=0.54(1)$, somewhat lower than in $\mathrm{CaC}_{6}$, but still in the intermediate coupling regime.

The difference $\Delta C_{p}$ between the normal and the superconducting state is shown in Fig. 2] At low temperatures $\Delta C_{p}(T)$ exceeds the BCS prediction while it is slightly lower than the BCS value near $T_{c}$. Using the ' $\alpha$-model' which assumes an isotropic $s$-wave BCS gap $\Delta(T)$ scaled by the factor $\alpha=\Delta(0) / k_{B} T_{c}$, we were able to fit the detailed temperature dependence of $\Delta C_{p}(T) / T$ by adjusting the gap ratio to $\alpha=1.67$. Accordingly, the corresponding $2 \Delta(0) / k_{B} T_{c}=3.34$ is somewhat reduced below the weak coupling BCS limit of 3.52 .

First, we discuss the anisotropy of the superconducting properties of $\mathrm{SrC}_{6}$. The anisotropy of the upper critical fields, $\Gamma_{H}=H_{c 2}^{\perp} / H_{c 2}^{\|}$amounts to $\approx 2$ at $T \sim T_{c} / 2$, close to that of $\mathrm{YbC}_{6}[1]$, but significantly smaller than found in $\mathrm{CaC}_{6}\left(\Gamma_{H} \sim 4\right)[2]$. Assuming an isotropic superconducting gap, the anisotropy $\Gamma_{H}$ reflects the anisotropy of the Fermi velocities. Our ab-initio calculations for the Fermi surface (FS) of $\mathrm{CaC}_{6}$ and $\mathrm{SrC}_{6}$ clearly reveal an elliptical sheet, associated mainly to interlayer states, and a tubular structure, of $\pi^{*}$ character ( $c f$. Ref. [16]). In

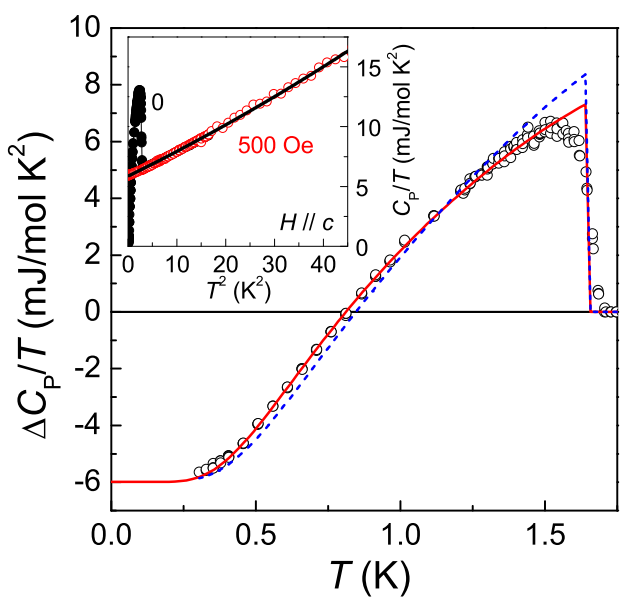

FIG. 2: (Color online) Temperature dependence of $\Delta C_{p} / T$ $=C_{p}(H=0) / T-C_{p}(H=500 \mathrm{Oe}) / T$. The (blue) dashed and (red) solid lines represent the BCS curve and the best fit according to the $\alpha$-model (see text), respectively. The inset shows the temperature dependence of $C_{p}$ at $H=0$ and 500 Oe. The solid (black) line through the data points for $H=$ 500 Oe is a fit to a polynomial as described in the text. 
$\mathrm{SrC}_{6}$ the FS for the IL bands has a more pronounced $2 \mathrm{D}$ character and is open, which results from the $10 \%$ increase of the $c$ axis lattice parameter as compared to $\mathrm{CaC}_{6}$. The anisotropy of the average Fermi velocities $\Gamma_{v_{F}}=\left(<v_{F_{a b}}^{2}>/\left\langle v_{F_{c}}^{2}\right\rangle\right)^{1 / 2}$ is very close in the two compounds: $\Gamma_{v_{F}} \approx 1.9$ in $\mathrm{CaC}_{6}$ and $\approx 1.7$ in $\mathrm{SrC}_{6}$. If we, however, consider only the IL sheets where the superconducting gap is larger [18], we find a much larger difference: $\Gamma_{v_{F}}=1.1$ for $\mathrm{CaC}_{6}$ and $\Gamma_{v_{F}}=2.1$ for $\mathrm{SrC}_{6}$. Therefore, $\Gamma_{H}$ of $\mathrm{SrC}_{6}$ is expected to be larger or at least similar to that of $\mathrm{CaC}_{6}$. The significantly enlarged anisotropy of $H_{c 2}$ in $\mathrm{CaC}_{6}$ with respect to $\mathrm{SrC}_{6}$ therefore cannot be understood in terms of the anisotropy of the Fermi velocities but must be attributed to an anisotropy of the superconducting gap as well.

This conclusion is supported by the reduced $C_{p}$ jump observed in $\mathrm{SrC}_{6}$. Assuming an isotropic gap, the normalized jump, $\Delta C_{p}\left(T_{c}\right) / \gamma_{N} T_{c}$, grows with the $e$-ph coupling strength over the BCS weak coupling limit of 1.432. With $\lambda \approx 0.54$ estimated from $C_{p}, \Delta C_{P}\left(T_{c}\right) / \gamma_{N} T_{c}$ is expected to be enhanced over the BCS value. The experimental $\Delta C_{p}\left(T_{c}\right) / \gamma_{N} T_{c}=1.426$, however, is smaller than the BCS value. As a characteristic feature of the anisotropic superconducting gap, the entropy "lost" near $T_{c}$ is transferred to lower temperatures (Fig. 2) [18]. These findings indicate that the superconducting gap in $\mathrm{CaC}_{6}$ as well as in $\mathrm{SrC}_{6}$ has a marked anisotropy, which is also supported by recent calculations [18], showing that $\mathrm{CaC}_{6}$ indeed exhibits a strongly $\mathbf{k}$-dependent superconducting gap due to anisotropic $e$-ph interaction. Considering that the deviation of $\Delta C_{p}(T)$ from the predicted curve for the isotropic gap model is less pronounced

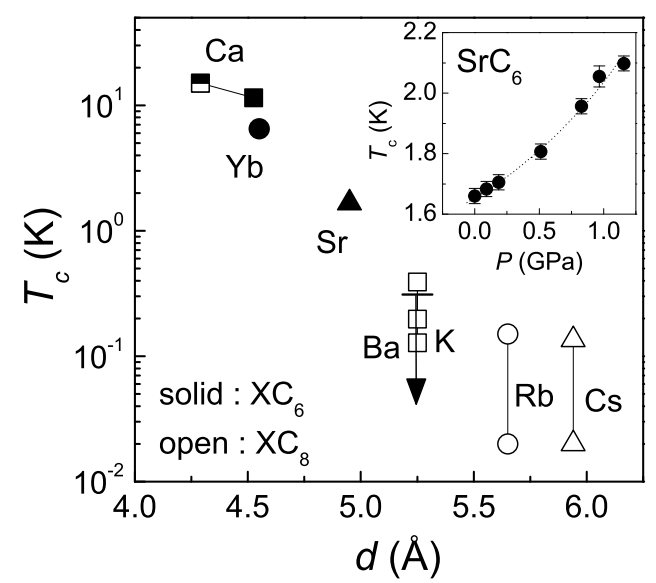

FIG. 3: $T_{c}$ as a function of the graphite layer distance, $d$ for the alkali-GICs, $X \mathrm{C}_{8}(X=\mathrm{K}, \mathrm{Rb}$ and $\mathrm{Cs})$ and the alkaline earth-GICs $X_{6}(X=\mathrm{Ca}, \mathrm{Yb}, \mathrm{Sr}$, and $\mathrm{Ba})$. For $\mathrm{CaC}_{6}, T_{c}$ at high pressure $(P=8 \mathrm{GPa})[10$ ] is also plotted (the halfshaded square) and the graphite layer distance for the compressed $\mathrm{CaC}_{6}$ is estimated from the theoretically calculated bulk modulus [9]. The upper limit of $T_{c}$ for $\mathrm{BaC}_{6}$ is indicated by the arrow. The inset shows $T_{c}$ vs. pressure for $\mathrm{SrC}_{6}$. for $\mathrm{SrC}_{6}$ than for $\mathrm{CaC}_{6}$ [17], the superconducting gap anisotropy is weaker for $\mathrm{SrC}_{6}$. This is also consistent with the reduced anisotropy in $H_{c 2}$. Therefore, replacing $\mathrm{Ca}$ with $\mathrm{Sr}$ decreases not only the strength of the $e$-ph coupling, but also its anisotropy.

We can now discuss the reduced $T_{c}$ of $\mathrm{SrC}_{6}$, which is about an order of magnitude smaller than $T_{c}$ of $\mathrm{CaC}_{6}$. When $\mathrm{Ca}$ is replaced with $\mathrm{Sr}, T_{c}$ decreases much more than it would be expected in the view of the alkalineearth "isotopic" substitution [4]. With $\sqrt{M_{\mathrm{Sr}} / M_{\mathrm{Ca}}} \simeq$ 1.48 , one would expect a $T_{c}$ of $7.8 \mathrm{~K}$ for $\mathrm{SrC}_{6}$, hence much larger than the experimental value. We thus conclude that the mass of the intercalant is not the main factor which determines $T_{c}$. Instead, we found that the $T_{c}$ 's of the superconducting GICs strongly depend on the graphite interlayer distance, $d$. Figure 3 illustrates that $T_{c}$ decreases rapidly, almost exponentially with increasing $d$ for both alkali- and alkaline earth-GICs 25, 26]. The increase of $T_{c}$ with pressure found for $\mathrm{CaC}_{6}[8,9,10$ and $\mathrm{SrC}_{6}\left(d T_{c} / d P \sim 0.35 \mathrm{~K} / \mathrm{GPa}\right.$, the inset of Fig. 3) clearly manifests a similar trend. Obviously, the main factor that governs the significant decrease of $T_{c}$ in $\mathrm{SrC}_{6}$ as well as the absence of superconductivity in $\mathrm{BaC}_{6}$ down to $0.3 \mathrm{~K}$, is the increased distance between the graphite layers.

Figure 4 clearly demonstrates why. In the calculated phonon density of states (PhDOS) (Fig. 廿( (c)) and the corresponding Eliashberg functions $\alpha^{2} F(\omega)$ (Fig. 4(d)) for $\mathrm{CaC}_{6}$ and $\mathrm{SrC}_{6}$, we observe three groups of phonons: intercalant-related vibrations $\left(I_{x y}\right.$ and $\left.I_{z}\right)$ at $\omega \leq 20 \mathrm{meV}, \mathrm{C}$ out-of-plane vibrations $\left(\mathrm{C}_{z}\right)$ around
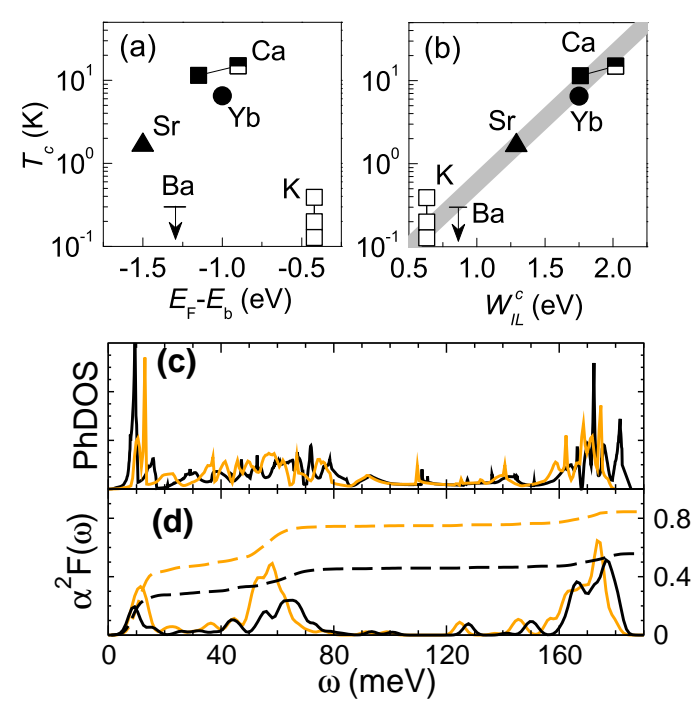

FIG. 4: (Color online) (a) $T_{c}$ vs. the position of the bottom of the interlayer bands $\left(E_{b}\right)$ with respect to $E_{F}$ (b) $T_{c}$ vs. the band width of the interlayer bands along the $c$ axis $\left(W_{I L}^{c}\right)$. The grey line is meant as guide to the eye. (c) Phonon density of states and (d) Eliashberg function $\alpha^{2} F(\Omega)$ and frequency-dependent electron-phonon coupling $\lambda(\omega)=$ $2 \int_{0}^{\omega} \alpha^{2} F(\Omega) / \Omega d \Omega$ for $\mathrm{CaC}_{6}$ (orange) and $\mathrm{SrC}_{6}$ (black). 
$50 \mathrm{meV}$, and $\mathrm{C}$ bond-stretching vibrations $\left(\mathrm{C}_{x y}\right)$ at $\omega>150 \mathrm{meV}$. The qualitative shape of $\alpha^{2} F(\omega)$ is the same in the two compounds, indicating a similar spectral distribution of the $e$-ph interaction. But the total $\lambda$ decreases from 0.83 in $\mathrm{CaC}_{6}$ to 0.56 in $\mathrm{SrC}_{6}$, while the logarithmic-averaged phonon frequency $\left(\omega_{l n}\right)$ remains unchanged at $\sim 305 \mathrm{~K}$. The results are in very good agreement with the previous calculations for $\mathrm{SrC}_{6}$ with the $\alpha \beta \gamma$ stacking [16]. Within computational accuracy, the difference between the $\alpha \beta$ and $\alpha \beta \gamma$ stackings seems negligible in contrast to a previous conjecture [4]. Using the Allen-Dynes formula, with $\mu^{*}=0.145$, we obtain $T_{c}=11.4 \mathrm{~K}$ for $\mathrm{CaC}_{6}$ and $T_{c}=3.1 \mathrm{~K}$ for $\mathrm{SrC}_{6}$, in reasonable agreement with the experimental results and previous calculations [16].

The reduction of $T_{c}$ in $\mathrm{SrC}_{6}$ is due to the simultaneous decrease of the $I_{x y}$ and $\mathrm{C}_{z}$ contribution to the $e$-ph coupling. The reduced coupling for the low-lying $I_{x y}$ vibrations has a negative effect on $T_{c}$, but it is also very effective in increasing $\omega_{l n}$, thus, the effect is partly compensated. On the other hand, the large reduction of coupling associated to $\mathrm{C}_{z}$ vibrations, which happens at energy scales comparable to $\omega_{l n}$, reduces $\lambda$ leaving $\omega_{l n}$ unchanged, and thus has a large effect on the $T_{c}$. The reduction of $e$-ph coupling for $\mathrm{C}_{z}$ vibrations in the intermediate energy range is essential to explain the significantly lower $T_{c}$ for $\mathrm{SrC}_{6}$ than $\mathrm{CaC}_{6}$.

The $e$-ph interaction for the $\mathrm{C}_{z}$ modes, and to a lesser extent for the $I_{x y}$ ones, is associated to the interband coupling between the IL and $\pi^{*}$ states [27]. Going from $\mathrm{CaC}_{6}$ to $\mathrm{SrC}_{6}$, this interband coupling is essentially reduced by the increase of the $c$ axis lattice constant, which decreases the real-space overlap between IL and $\pi^{*}$ wavefunctions. The characteristic parameter monitoring the IL- $\pi^{*}$ overlap is the bandwidth of the IL band along the $c$ axis $\left(W_{I L}^{c}\right)$. Figure $4(\mathrm{~b})$ demonstrates that $T_{c}$ for both alkali- and alkaline earth-GICs is directly correlated to $W_{I L}^{c}$, clearly explaining the $T_{c}$ dependence on the graphite layer distance. This observation is in strong contrast to the dependence of $T_{c}$ on the degree of filling for the IL band (Fig. 廿(a)). Even though this band has to be occupied for superconductivity to occur [3], there is no clear correlation of $T_{c}$ with the number of IL electrons.

Our findings indicate that $\mathrm{C}$ phonon modes play a nontrivial role in the superconductivity of the GICs, in contrast with the conjecture from the $\mathrm{Ca}$ isotope experiments [12]. Since C would give a sizable contribution to the isotope effect, the total isotope exponent would exceed the BCS limit $\alpha_{T_{c}}=0.5$, which requires further studies on the effects of $\mathrm{C}$ isotope substitution.

In conclusion, we reported that $\mathrm{SrC}_{6}$ becomes superconducting at $T_{c}=1.65(6) \mathrm{K}$, but $\mathrm{BaC}_{6}$ stays normal conducting down to $0.3 \mathrm{~K}$. The reduced $C_{p}$ jump at $T_{c}$ in $\mathrm{SrC}_{6}$ and the reduced anisotropy $H_{c 2}$ in comparison with the corresponding data for $\mathrm{CaC}_{6}$ strongly supports the idea of an anisotropic superconducting gap [17, 18].
We give clear evidence that $T_{c}$ of the GICs essentially depends on the graphite layer distance due to sensitive change of $e$-ph coupling for both in-plane intercalant and the out-of-plane $\mathrm{C}$ phonon modes. Our results suggest that a possible route to increase $T_{c}$ is to replace Ca by smaller atoms, such as Mg. Several attempts to prepare pure $\mathrm{MgC}_{6}$ have failed so far, but partial substitution of $\mathrm{Mg}$ or Li, as long as the filling of the IL bands is kept, could be possible to reduce $d$, stabilize the structure, and as a result, increase $T_{c}$.

The authors acknowledge useful discussion with A. Simon, O. K. Andersen, G. B. Bachelet, M. Giantomassi and D. Guerald, and we thank E. Brücher, S. Höhn for experimental assistance.

[1] T. E. Weller, M. Ellerby, S. S. Saxena, R. P. Smith, and N. T. Skipper, Nat. Phys. 1, 39 (2005).

[2] N. Emery, et al., Phys. Rev. Lett. 95, 087003 (2005).

[3] G. Csányi, P. B. Littlewood, A. H. Nevidomskyy, C. J. Pickard and B. D. Simons, Nat. Phys. 1, 42 (2005).

[4] I. I. Mazin, Phys. Rev. Lett. 95, 227001 (2005).

[5] M. Calandra and F. Mauri, Phys. Rev. Lett. 95, 237002 (2005).

[6] G. Lamura, et al., Phys. Rev. Lett. 96, 107008 (2006).

[7] J. S. Kim, R. K. Kremer, L. Boeri, and F. S. Razavi, Phys. Rev. Lett. 96, 217002 (2006).

[8] R. P. Smith et al., Phys. Rev. B 74, 024505 (2006).

[9] J. S. Kim, L. Boeri, R. K. Kremer, and F. S. Razavi, Phys. Rev. B. 74, 214513 (2006).

[10] A. Gauzzi et al., Phys. Rev. Lett. 98, 067002 (2007).

[11] N. Bergeal et al., Phys. Rev. Lett. 97, 077003 (2006).

[12] D. G. Hinks, D. Rosenmann, H. Claus, M. S. Bailey, and J. D. Jorgensen, Phys. Rev. B 75, 014509 (2007).

[13] S. Sanfilippo et al., Phys. Rev. B 61, R3800 (2000); G. Satta, G. Profeta, F. Bernardini, A. Continenza, and S. Massidda, ibid. 64, 104507 (2001).

[14] I. I. Mazin and D. A. Papaconstantopoulos, Phys. Rev. B 69, 180512(R) (2004); M. Giantomassi, L. Boeri, and G. B. Bachelet, ibid. 72, 224512 (2005).

[15] A. N. Kolmogorov and S. Curtarolo, Phys. Rev. B 73, 180501(R) (2006); A. Y. Liu and I. I. Mazin, ibid. 75, 064510 (2007).

[16] M. Calandra and F. Mauri, Phys. Rev. B 74, 094507 (2006).

[17] I. I. Mazin et al., condmat/0606404.

[18] A. Sanna et al., Phys. Rev. B 75, 020511(R) (2007).

[19] D. Guerard, M. Chaabouni, P. Lagrange, M. El Makrini, and A. Hérold, Carbon 18, 257 (1980).

[20] S. Baroni, et al. URL http://www.pwscf.org.

[21] For computational details, see Ref. [9]. For Sr, we used an ultrasoft [22] pseudopotential, with semicore $s$ and $p$ states treated as valence.

[22] D. Vanderbilt, Phys. Rev. B 41, R7892 (1990).

[23] N. R. Werthammer, E. Helfand, and P. C. Hohenberg, Phys. Rev. 147, 295 (1966).

[24] Y. Koike, H. Suematsu, K. Higuchi, and S. Tanuma, Physica B 99, 503 (1980).

[25] M. S. Dresselhaus and G. Dresselhaus, Adv. Phys. 51, 1 
(2002).

[26] Here we do not consider the metal-rich GICs synthesized under high pressures such as $\mathrm{LiC}_{2}$ which are known to be metastable.
[27] L. Boeri, G. B. Bachelet, M. Giantomassi, and O. K. Andersen, condmat/0701347. 